\title{
La mujer latinoamericana dentro del contexto de los Derechos Humanos: énfasis desde la perspectiva del derecho al trabajo*
}

\author{
Yaneth Vargas Sandoval* \\ Carla Rita Bracchi Silveira
}

Recibido: 20 de enero de 2016 • Revisado: 7 de febrero de 2016

Aprobado: 22 de febrero de 2016

\section{Resumen}

Se analizará en el presente documento el papel de la mujer dentro de la sociedad y específicamente dentro del poder acceder a un trabajo. Para el efecto, se iniciará con el estudio de los tratados y convenios de derechos humanos que reglamentan los derechos de las mujeres en cuanto a la protección en condiciones de igualdad. Lo anterior en razón a que es importante abordar académicamente la realidad iberoamericana en el contexto de la vinculación laboral de las mujeres.

Palabras clave: Derecho al trabajo, mujeres, Latinoamérica.

" El presente artículo es producto del proyecto de investigación institucional de la Universidad de Bahia de Brasil: "La Seguridad Social: un derecho humano en desarrollo". El citado proyecto es financiado por la mencionada universidad.

* Abogada Universidad Libre - Colombia. Especialista en Derecho procesal - Universidad Libre. Especialista en Docencia Universitaria - Universidad la Gran Colombia. Magister en Dirección y Gestión de los Sistemas de Sistemas de Seguridad Social en la Universidad Alcalá Henares- OISS. Madrid - España. Docente de la Universidad la Gran Colombia- Universidad Libre- Bogotá- Colombia. Correo electrónico: yanvargass@hotmail.com.

* Abogada de la Universidad Estadual de Santa Cruz- Brasil. Especialista en Derecho Material y Procesal del Trabajo de la Facultad de Ciencias y Tecnología de Itabuña- Brasil. Especialista en Derecho Ambiental de la Pontificia Universidad Católica de Minas Gerais. Magister en Derecho del Trabajo y relaciones internacionales de la Universidad Nacional Tres de Febrero de Argentina. Docente de la Universidad de Bahía - Facultad Madre Thais-FMT- Bahía - Brasil. Correo electrónico: carlabracchi@ uol.com.br. 


\title{
Latin American women in the context of Human Rights: EMPHASIS FROM THE PERSPECTIVE OF THE Right TO WORK
}

\begin{abstract}
The role of women in society and specifically within to access a job, be analyzed in this paper. To this end, it will start with the study of treaties and human rights conventions that regulate the rights of women in terms of equal protection. This is due to address the Iberoamericana academically important reality in the context of the employment relationship of women.
\end{abstract}

Keywords: Right to Work, Women, Latin America.

\section{A mulher latino-Americana no contexto dos Direitos Humanos: ÊNFASE a PARTIR DA PERSPECTIVA Do Direito AO TRABALHO}

\section{Resumo}

Analisar-se-á no presente documento o papel das mulheres na sociedade e, especificamente, dentro do poder acessar para um trabalho. Para este fim, vai-se começar com o estudo de tratados e convenções de direitos humanos que regulam os direitos das mulheres em termos de proteção em condições de igualdade. Isto porque é importante abordar academicamente a realidade ibero-americana no contexto da ligação de emprego das mulheres.

Palavras-chave: Direito ao Trabalho, Mulheres, Latino-americana.

\section{Introducción}

Para la premio Nobel de la Paz, la guatemalteca Rigoberta Menchu expresó: "Una mujer con imaginación es una mujer que no solo sabe proyectar la vida de una familia y la de una sociedad, sino también el futuro de un milenio" (2000, p. 4), pero como proyectar un futuro sin discriminación, y en el que la mujer ocupe el lugar que le corresponde en términos de igualdad; En el 2015 y después de 70 años de la reglamentación de los derechos humanos de la mujer por parte de la 
ONU, se sigue con el mismo objetivo, intentar erradicar la desigualdad entre los hombres y las mujeres.

En Latinoamérica, uno de los graves problemas en cuanto a la realidad laboral se presenta en la informalidad de los empleos, realidad que se hace más evidente entre las mujeres latinoamericanas, ya que no pueden acceder fácilmente a un trabajo formal. Lo que las pone en la informalidad o el desempleo, donde se le vulneran sus mínimos derechos laborales, en la informalidad los salarios son aún más bajos que el mismo salario mínimo de los respectivos países. Otro aspecto a tener cuenta es el hecho que existen trabajos de alto contenido de género como el servicio doméstico, en el cual la vulneración de los derechos de las mujeres es evidente aun existiendo regulación para su protección, claro ejemplo de ello es que casi 14\% de las trabajadoras domésticas reciben menos del salario mínimo legal por hora (CEPAL, FAO, ONU Mujeres, PNUD y OIT, 2013). O las cuidadoras a quienes no se les reconoce salario, porque suelen confundirse con responsabilidades familiares, propias de mujeres.

\section{Derechos humanos de las mujeres dentro de los tratados internacionales de derechos humanos}

Los derechos humanos, como forma de protección de todo el género humano, han tenido que reflexionar sobre aspectos muy puntuales de algunas personas, como las mujeres que han requerido de una protección significativa, por el contexto histórico y social en el que se han desarrollado sus derechos.

El primer documento que plantea la igualdad entre hombres y mujeres, y su no discriminación será el documento fundador de la Organización de Naciones Unidas, la Declaración Universal de los Derechos Humanos (1945), donde se plantea la igualdad de género, en ella se determina que debe existir igualdad entre hombres y mujeres, de la misma manera que se debe propender por la promoción $\mathrm{y}$ fomento de los derechos humanos para las mujeres.

Posteriormente en el Pacto Internacional de Derechos Civiles y Políticos en el artículo 3, se determina que se debe asegurar a los hombres y a las mujeres igual título a gozar de todos los derechos enunciados en el tratado. Igual manifestación se desprende del artículo 3 del Pacto de Derechos Económicos, Sociales y Culturales.

El reconocimiento de los derechos de la mujer fue más allá, en 1981 con la Convención sobre la Eliminación de todas las formas de Discriminación contra la 
Mujer (CEDAW por sus siglas en inglés), constituyéndose en la carta de derechos de la mujer, documento que consolidó un programa para ponerle fin a la discriminación. Para hacer revisión de esta Convención se hizo necesaria la creación del comité para la eliminación de la discriminación contra la mujer.

Para los años noventa aún persistía la discriminación de la mujer, por lo que las Naciones Unidas en 1994 plantearon la creación de un relator especial encargado de investigar las causas y consecuencias de la violencia contra las mujeres. Unido a este, se creó en el 2010, por parte del Consejo de Derechos Humanos, un Grupo de Trabajo sobre cuestiones de discriminación contra la mujer en la legislación y en la práctica.

En 1995 se realiza en Beijing la Cuarta Conferencia Mundial sobre la Mujer, en esta se adoptó una Plataforma de Acción que establece 12 esferas críticas para el adelanto de las mujeres y fue aprobada por unanimidad por los Estados Miembros de la ONU.

Para el 2001 se sigue hablando de la igualdad de género, esta vez en Durban, en la Conferencia Mundial contra el racismo, la discriminación, la xenofobia y las formas conexas de intolerancia, se plantearon que los derechos de las mujeres, son esenciales para el desarrollo de las sociedades. Haciendo énfasis en la no discriminación en el lugar de trabajo y asegurar la plena igualdad en la legislación laboral (CEPAL, FAO, ONU Mujeres, PNUD y OIT, 2013).

Pero una de las declaraciones más importantes respecto de los derechos humanos fue la celebrada el 8 de septiembre del 2000, Declaración del Milenio con la cual se reafirma la razón de ser de la ONU, se establecieron ocho objetivos de desarrollo interrelacionados en una agenda global, a los cuales se les denominó Objetivos de Desarrollo del Milenio (ODM), que han sido asumidos por todos los países del mundo.

\subsection{Derechos humanos a nivel regional}

A nivel regional, igualmente se han realizado ingentes esfuerzos en pro de los derechos humanos, entre los que se encuentran la Organización de Estados Americanos- OEA, organismo que estableció en 1928 la Comisión Interamericana de Mujeres - CIM, creado para la protección de los derechos de las mujeres y la igualdad de género en las Américas (OEA, 2015).

En 1998 se elabora por la Comisión Interamericana de Mujeres, el Programa Interamericano sobre la Promoción de los Derechos de la Mujer y la Equidad 
e Igualdad de Género, con este se pretende que los estados partes de la OEA, formulen políticas públicas, estrategias y propuestas para la promoción de los derechos humanos de la mujer y la igualdad de género en todas las esferas de la vida pública y privada. Asimismo pretende el acceso de la mujer al trabajo y a los recursos productivos en condiciones de igualdad.

\subsection{Organización Internacional del Trabajo y derechos del trabajo de las mujeres}

Desde 1919, con la creación de la Organización Internacional del TrabajoOIT, se establece la igualdad de derechos laborales para las mujeres y hombres, considerado como un derecho humano el trabajo en condiciones dignas. La OIT ha emitido convenios específicos para la no discriminación de la mujer entre los que se encuentran el Convenio número 100 y 122 sobre igualdad de remuneración en 1951, el Convenio número 111 sobre la discriminación (empleo y ocupación) en 1958, el Convenio número 156 sobre trabajadores con responsabilidades familiares de 1981, el Convenio número 103 de 1952 y el convenio número 103 del 2000 sobre protección de la maternidad (OIT, 2015a).

Para el 2010-2015 la OIT estableció un Plan de acción sobre la igualdad de género, promoviendo dos líneas de acción: la primera será analizar las necesidades específicas de hombres y mujeres en todas las iniciativas que de igualdad se lleven a cabo, y realizar intervenciones dirigidas a grupos específicos con el fin de lograr la participación tanto de mujeres como de hombres, para conseguir que se beneficien por igual de los esfuerzos por alcanzar el desarrollo (OIT, 2015a).

Respecto de la igualdad de género y la eliminación de la discriminación como objetivo transversal relacionado con el crecimiento económico con generación de empleo para hombres y mujeres y la efectiva aplicación de los derechos y principios fundamentales en el trabajo aprobó la Agenda Hemisférica de Trabajo Decente de las Américas, aprobada en la XVI reunión Americana de la OIT en Brasilia en 2006 (CEPAL, FAO, ONU Mujeres, PNUD y OIT, 2013).

Uno de los documentos más ambiciosos generados de las Naciones Unidas, son los denominados Objetivos de Desarrollo del Milenio (ODM), son propósitos para el desarrollo humano, fijados en el 2000 para ser cumplidos al 2015, determinado en el objetivo $3^{\circ}$. Promover la igualdad entre los géneros y la autonomía de la mujer.

Estos son algunos de los tratados sobre derechos humanos que se han establecido en pro de la igualdad entre las mujeres y los hombres, pero serán abordados 
en la segunda parte de este documento al tratar la situación real que enfrentan las mujeres en Iberoamérica en cuanto al acceso al trabajo.

\section{Vinculación de la mujer al trabajo decente en Latinoamérica}

\subsection{Mujer y trabajo}

El empleo es la forma en que las mujeres pueden alcanzar la autonomía económica y salir de la pobreza, es la única forma en que pueden generar sus propios recursos y no depender de otros, desligándose de manera efectiva su condición de dependencia (CEPAL, FAO, ONU Mujeres, PNUD y OIT, 2013).

Pero la mujer no debe acceder a cualquier trabajo, lo fundamental es que accedan a un trabajo dentro de los lineamientos de un trabajo decente. Que según el planteamiento de la OIT es un trabajo que sea productivo y que produzca un ingreso digno, seguridad en el lugar de trabajo y protección social para las familias, mejores perspectivas de desarrollo personal e integración a la sociedad, libertad para que la gente exprese sus opiniones, organización y participación en las decisiones que afectan sus vidas, e igualdad de oportunidad y trato para todas las mujeres y hombres (OIT, 2015b).

No se debe desconocer que la vinculación de la mujer al trabajo formal ha aumentado en los últimos años, para el año 2000 estaba en el 49.2\% y para el 2010 llega al 52.65\%, mientras que en los hombres cayó un punto siendo para el 2000 del $80.8 \%$ y en el 2010 del 79.6\%. Aun así la brecha laboral entre hombre y mujeres es elevada en el acceso al trabajo.

En lo que respecta a los países de América Latina, Perú y Bolivia, son los que registran mayor tasa de participación laboral femenina. Brasil, Colombia, Paraguay y Uruguay tienen un promedio del 52.6\%, siendo un promedio intermedio en la región, en tanto que la tasa más baja de participación laboral de la mujer se encuentra en Chile, Costa Rica, Honduras y México, con tasas inferiores a 45\% (CEPAL, FAO, ONU Mujeres, PNUD y OIT, 2013).

Este trabajo formalizado implica que sean reconocidos todos los derechos laborales y de seguridad social. Pero aun así, no existe una igualdad real, ya que los salarios para las mujeres son inferiores. De acuerdo al Informe Mundial sobre salarios 2014/2015 de la OIT, el promedio de salarios para las mujeres es inferior 
del 4\% y 36\% al de los hombres, aumentándose esta brecha salarial cuando las mujeres están más capacitadas.

\subsection{Situación de la mujer dentro del trabajo precario}

Otro aspecto importante para tener en cuenta es el desempleo, para la OIT, en el 2015, la desaceleración económica en Latinoamérica causa un aumento del desempleo e indicios de aumento en la informalidad. Presentándose una mayor incidencia en las mujeres y jóvenes, y podría aumentar para el 2016. La cantidad de personas que para el 2015 se encuentran buscando empleo son al menos 1.7 millones (OIT, 2015d).

En América Latina y el Caribe, si bien es cierto se ha reducido el desempleo, el mayor problema que se afronta es el trabajo informal, hay 130 millones de personas ocupadas que tienen empleos informales, sin protección social ni derechos laborales (OIT, 2014).

Pero son las mujeres quienes sufren más las consecuencias del trabajo informal, según el Banco Mundial, la mitad de los trabajadores latinoamericanos tienen un empleo informal, el 45\% de los hombres y el 50\% de las mujeres que trabajan en condición de informalidad, lo que coloca a la mujer en un estado de vulnerabilidad, ya que no puede acceder a un trabajo decente (Banco Mundial, 2014).

En América Latina, esta brecha laboral en detrimento de las mujeres se observa en países como Nicaragua, Bolivia, Perú y Colombia. En estos países, respectivamente, el 59.4\%, 54.3\%, 51\% y 50.9\% de las mujeres trabajan en trabajos precarios (CEPAL, FAO, ONU Mujeres, PNUD y OIT, 2013). Entre los factores que llevan a que las mujeres se empleen en la informalidad, se encuentran: la falta de educación, la pobreza, o el no poder conciliar su relación familiar y trabajo, entre otros.

\subsubsection{Trabajo de las mujeres en el servicio domestico}

El acceso al trabajo de las mujeres con menor nivel educativo y en situación de pobreza es la vinculación en trabajos de servicio doméstico. Este trabajo se caracteriza por desarrollar un sin número de actividades para las familias que no son propias. El trabajo en servicio doméstico no ha sido lo suficientemente valorado, siendo una de las actividades donde se encuentra el mayor carencia de trabajo decente.

Aun cuando ha sido compleja la determinación de cuántas son las personas que laboran en actividades del servicio doméstico, ya que muchas de ellas son 
migrantes indocumentadas o se identifican como familiares cercanos de la familia donde labora, o laboran por horas; La OIT, para el 2011, estableció que laboraban en el servicio doméstico 19.5 millones de trabajadores en la región, de los cuales 18 millones son mujeres y 1.5 millones son hombres, siendo el 7\% de la ocupación urbana de la región (Valenzuela, 2012).

Lo anterior evidencia que es un trabajo desarrollado en su gran mayoría por mujeres, quienes ven en este trabajo la forma de entrar al mercado laboral. Pero son las mujeres más pobres, con un menor nivel de educación y que viven en un entorno de mayor exclusión social, quienes asumen que este trabajo en muchos casos no cumple con los parámetros de trabajo decente.

Ya que se trata de un trabajo que por mucho tiempo ha permanecido sin una adecuada formalización, en Latinoamérica algunos países aún sigue sin formalizarlo, y en los países donde esta formalizado es alto el nivel de incumplimiento de las normas sobre trabajo doméstico. Lo anterior lleva a que la cobertura en seguridad social de estas trabajadoras sea casi nula. Y por otro lado, el nivel salarial es el más bajo dentro de la escala laboral (Valenzuela, 2012).

\subsubsection{Trabajo de mujeres cuidadoras}

La división sexual del trabajo ha llevado a que las mujeres asuman ciertos trabajos que se muchas veces no son remunerados, dentro de los que se encuentran el trabajo dentro del hogar, el cuidado de niños, adultos mayores o enfermos, en estos trabajos las mujeres invierten la mayor cantidad de su tiempo, y al no ser remunerado, se ponen en situación de subordinación y dependencia de los hombres (CEPAL, FAO, ONU Mujeres, PNUD y OIT, 2013).

Este trabajo no remunerado es desarrollado fundamentalmente por la mujer, $\mathrm{y}$ no existen políticas públicas claras al respecto en Iberoamérica. Ya se están tomando medidas de apoyo para las cuidadoras, países como Chile y Ecuador son los únicos dos países en Latinoamérica que tienen transferencias para apoyar a los cuidadores de personas con discapacidad. Brasil, Costa Rica y Uruguay, por su parte, tienen servicios de inserción familiar para personas mayores y solo Costa Rica entrega recursos para cuidadores en fase terminal. Chile y Cuba cuentan con respiro para cuidadoras de adultos mayores. Y México y Argentina tienen programas de atención psicológica a cuidadoras. Uruguay tiene un programa integral de apoyo a todas las personas proveedoras de cuidados, tanto de forma remunerada como no remunerada (CEPAL, 2015). 
La OIT evidenció la situación de las cuidadoras por lo que emitió el convenio número 156 de 1981, sobre trabajadores con responsabilidades familiares, convenio que hasta el momento solo ha sido ratificado por 11 países de la región. Sin embargo, este convenio si bien es cierto establece las responsabilidades de ambos sexos en cuanto a la familia, reforzó el criterio de cuidadora de la mujer, como principal responsable del cuidado de la familia (OIT, 1981).

Otro aspecto a tener en cuenta es que muchas de las cuidadoras desarrollan labores dentro de sus propios hogares, lo que hace que su trabajo se visibilice aún más.

\section{Consecuencias de que la mujer no acceda a un trabajo decente}

\subsection{Brecha salarial}

Acceder a un trabajo formal a las mujeres les permite acceder a la garantía de un salario que le permita adquirir una calidad de vida digna, con un ingreso que le permita sobrevivir, pero aun teniendo un trabajo formal, el hecho de ser mujer le condiciona a no tener un salario igual al que puede percibir un hombre por la misma cantidad y calidad de trabajo.

Factores que generan que exista una brecha salarial entre mujeres y hombres son: infravaloración del trabajo de la mujer, características del puesto de trabajo, segregación basada en el sexo, estructura salarial dada por los Estados, ya que pueden fijar el salario dependiendo de sectores con predominio de valores, percibir a la mujer como dependiente, o la probabilidad de que las mujeres estén en sectores menos organizados y el modelo comportamental familiar también puede influir en la disparidad (OIT, 2015c).

Esta brecha salarial ha llegado a condicionar muchas veces el contexto de la maternidad, del Informe Mundial de Salarios 2014/2015 de la OIT se analizó que una de las condiciones para la diferencia salarial entre mujeres y hombres era la condición de ser o no madre. Planteándose como elementos sociológicos de dicha situación que la mujer no podía conciliar la vida familiar y laboral; Insuficientes guarderías y ocupaciones de las mujeres con menor retribución al emplearse en trabajos flexibles.

Otro aspecto que agrava la situación de inequidad de las mujeres es el trabajo realizado en trabajos informales o trabajadoras del servicio doméstico, ya que 
dichos trabajos no tienen remuneraciones adecuadas, siendo los salarios muchas veces inferiores a los mínimos establecidos en cada país. Pero también agrava la situación el trabajo no remunerado, como el desarrollado por las mujeres cuidadoras, aun cuando aporta de una manera importante dentro de la estructura del Estado.

\subsection{Seguridad social en pensión de vejez}

Las consecuencias de un empleo sin condiciones dignas, sin salarios adecuados o el no acceso al mismo, generan graves consecuencias en el sistema de pensiones, ya que en Latinoamérica, las pensiones de vejez son principalmente contributivas y van ligadas necesariamente a trabajos formales, razón por lo que para las mujeres el acceso a las mismas no es fácil.

En los países Iberoamericanos, las mujeres mayores perciben pensiones de inferiores cuantías a las de los hombres, muchas veces esto ocurre porque se vinculan más tarde al mundo laboral, o porque se emplean en actividades no formales o que no tienen protección en seguridad social (OISS, 2014b).

En Latinoamérica aproximadamente 130 millones de trabajadores no contribuyeron durante el 2010 a un esquema de pensiones para la vejez. Países como Bolivia, Guatemala, Honduras, Nicaragua, Paraguay y Perú, menos del 20\% de la fuerza laboral realizaron contribuciones. Colombia, República Dominicana y México, solamente entre un 30\% y un 40\% de los trabajadores contribuyen para pensiones. Y por último, Argentina, Brasil, Chile, Costa Rica, Panamá y Uruguay, aportan entre un $50 \%$ y un $70 \%$. Lo que evidencia que no todos podrán acceder a un derecho pensional de vejez (OCDE, BID y Banco Mundial, 2015).

En cuanto a las mujeres, ellas tienen una menor cobertura de seguridad social que los hombres; el 40.7\% de las mujeres ocupadas tienen trabajados sin protección, porcentaje que disminuye. Entre los países donde más se evidencia esta falta de protección se encuentran Paraguay, Perú, Colombia y México.

En Iberoamérica, los sistemas de pensiones se organizan en sistemas de reparto y sistemas de capitalización, en los cuales se establece una diferencia de edades para el reconocimiento de la pensión de vejez, esta diferencia ha sido defendida por algunos movimientos de mujeres en Latinoamérica, pero esta diferencia no es del todo un beneficio ya que en los sistemas de capitalización individual, la diferencia de edad, intensifica las desventajas de las mujeres al momento de reconocerse la pensión de vejez, y el valor de la misma. 
Y por otro lado, en el sistema de seguridad social en pensiones de reparto, la pensión está fuertemente ligada al trabajo formal; Y como se evidenció, las mujeres laboran en trabajos no formalizados y sin garantías de seguridad social. Lo que hace que las mujeres no accedan a esta clase de pensiones. Bajo estos lineamientos a la pensión que pueden acceder las mujeres es a la pensión de viudez, la cual recibirán siempre que celebre un contrato matrimonial o mantenga una convivencia con su esposo o compañero permanente, lo que la lleva a recibir como beneficio no autónomo sino ligado a estas situaciones jurídicas, considerándose así a la mujer subordinada a la dependencia de un vínculo matrimonial (Jiménez, s.f).

\subsection{Mujer en la vejez y pobreza}

La pobreza en la vejez es una cuestión de género, por cuanto la esperanza de vida de las mujeres es mayor, de los 53 millones de adultos mayores que viven en América Latina 29 millones son mujeres, lo que representa el 55\% del total de los mayores de la región (OISS, 2014a).

Pero la discriminación de la mujer en la vejez persiste, de acuerdo a la Recomendación general No.27 sobre las mujeres de edad y la protección de sus derechos humanos, de 16 de diciembre de 2010, del Comité para la Eliminación de la Discriminación contra la Mujer (CEDAW) se evidencia en que:

11. Si bien tanto el hombre como la mujer son objeto de discriminación a medida que envejecen, las mujeres viven el envejecimiento de distinta forma. El efecto de las desigualdades de género a lo largo de la vida se agrava con la vejez y con frecuencia se basa en normas culturales y sociales hondamente arraigadas. La discriminación que sufren las mujeres de edad suele ser el resultado de una distribución injusta de recursos, malos tratos, abandono y restricción del acceso a servicios básicos. (CEDAW, 2010)

La misma recomendación plantea que las mujeres son más longevas que los hombres según esta existen 83 hombres por cada 100 mujeres mayores de 60 años y, entre los mayores de 80 años, solo hay 59 hombres por cada 100 mujeres de más de 80 años. Pero esto también es contraproducente ya que las pensiones de viudez son económicamente más bajas que las de vejez (OISS, 2014b). 
Al tener menos ingresos o no tener ninguno, lleva a que las mujeres en la vejez dependan de sus familias o deban seguir empleándose o caigan en la indigencia, situación que evidencia que la pobreza también es un problema de género.

\subsection{Evidencia de discriminación de la mujer en Brasil}

Debido a la importancia que tiene Brasil dentro del contexto Latinoamericano se hace necesario hacer un acercamiento a la realidad de la mujer en Brasil. Las mujeres brasileñas son responsables de los medios de vida de aproximadamente el $37,3 \%$ de los hogares, el número demuestra la extrema relevancia social que tiene la mujer. Sin embargo, las mujeres tienen salarios que son hasta un $25 \%$ y un $30 \%$ menor que los del hombre.

Esta discriminación se presenta no solo cuanto al salario, sino que la discriminación se presenta en las relaciones laborales violentando el principio de igualdad entre las personas y el principio de la dignidad humana. Como principios básicos del sistema jurídico brasileño, el Estado ha desarrollado mecanismos que pretenden definir las formas de discriminación en las relaciones laborales.

\subsubsection{Marco normativo de no discriminación}

En la Constitución Federal de 1988, se plantean artículos basados en el principio de legalidad, que pretenden el reconocimiento de los derechos fundamentales inherentes a la persona humana, garantizando la igualdad entre las personas, y que prohíbe toda distinción o discriminación de etnia, raza, color o credo.

$\mathrm{El}$ derecho constitucional de Brasil se fundamenta en un país democrático de derecho, teniendo en cuenta su base los principios de legalidad, igualdad y dignidad humana. Igualmente la Ley federal No 9029 del 13 de abril de 1995, se ocupa de las formas de discriminación en las relaciones laborales, con lo que plantea casos de alerta en el que se pueden configurar la práctica discriminatoria en las relaciones laborales y el empleo.

Es importante para el análisis de las disposiciones legales, doctrinas y jurisprudencia sobre el tema, definir qué se debe entenderse por discriminación, ya que la idea del legislador es promover a través de la institución legalista promoción de la igualdad y las condiciones de las oportunidades de empleo o de empleo para todos los ciudadanos, corroborando la erradicación y la exclusión de cualquier instituto o las formas de discriminación de los ciudadanos brasileños. 
Lima (2011) habla de la falta de conceptualización dentro de la norma, de la terminología “discriminación”, que apunta a la legislación relativa a los actos discriminatorios y no como la definición y el significado del término. Señala que la Ley que define los tipos de discriminación, la Ley No Federal 9029/1995 no hace mención de su conceptualización y la Ley No 9799/1999 es la que plantea específicamente la discriminación contra las mujeres.

Discriminación desde el punto de vista conceptual es aquella conducta que se exterioriza a través de la manifestación de actos que no permiten la calificación en el sentido positivo de los hechos, no dar al individuo a lo que sería el término correcto de la situación presentada, es decir "La discriminación es la conducta por la cual la persona se niega, directamente los criterios de manera injusta descalificación, el tratamiento compatible con el establecimiento de la norma legal para la situación concreta para que experimentö" (Delgado, 2010, p. 725).

\subsubsection{Principio de no discriminación en las relaciones laborales}

El principio de no discriminación es una especie de principio de igualdad, que tiene por objeto garantizar que no haya discriminación en las relaciones laborales. El principio de igualdad, a su vez, tiene amplitud y ofertas con el tratamiento de la garantía de igualdad a nivel mundial, ya que el principio de no discriminación se orienta con la Ley del Trabajo, con el fin de proteger al empleado en las relaciones laborales, puesto que la naturaleza del empleo ya apunta a la diferenciación económica entre las partes por la superioridad económica del empleador y el empleado por su inferioridad.

Sin embargo, la no discriminación no es una tarea fácil, ya que la ideología es respetar las diferencias, porque la humanidad es diversa en la raza, el color, la religión, las creencias y culturas y como era para Aristóteles, la igualdad es para tratar la misma como iguales y desiguales como desiguales, que muestran la complejidad de este tema.

El principio de no discriminación sirve para cumplir uno de los pilares estratégicos de la Ley del Trabajo, es el respeto de las normas internacionales del trabajo. En este sentido, las reglas observadas en el sistema legal adoptan la convención dictada por la Organización Internacional del Trabajo, y se basan en la regulación de las formas de controlar el comportamiento humano en la vida en la sociedad, por lo tanto, la misma regulación dirige a los principios aplicables. 
Las disposiciones legales que apoyan la no discriminación en las relaciones laborales en Brasil, por cuestiones de género, se ubican en la Constitución Federal, en varios dispositivos legales como en la Ley N 9.029 / 1995 y en el Convenio 111 de la Organización Internacional del Trabajo (OIT).

\subsubsection{Discriminación de la mujer en el ámbito laboral en Brasil}

En el artículo 2 de la Ley Federal No 9.029 / 1995 se establece la determinación de la no discriminación en las relaciones laborales, situación que pone de relieve los viejos problemas, los prejuicios y la discriminación hacia las mujeres. La mujer está sujeta a cargas sociales, producto del diseño social y de aspectos inherentes a los condicionamientos fisiológicos como la maternidad. Aspectos que han tenido que ser protegidos a través de la jurisprudencia de los Tribunales Judiciales brasileros, donde en los últimos años se han protegido los derechos y garantías de las mujeres.

Aspectos como el despido sin causa por encontrarse en estado de embarazo, o la discriminación en el proceso de selección, al ser requisito para acceder al cargo en las empresas prueba de embarazo o de control de la natalidad, o cuando se despide a la trabajadora, por el conocimiento de la empresa del estado de embarazo, han tenido que ser protegidos a través de fallos judiciales.

El fundamento tenido en cuenta por los Tribunales ha sido que estos aspectos, violentan el derecho a la intimidad de las mujeres, derecho que es protegido en la Constitución de 1988, lo que lleva a que se sancionen a los empresarios, con la pena de readmisión y conceder a las mujeres el derecho a reclamar daños y perjuicios.

En la jurisprudencia del Tribunal Regional del Trabajo de la 3a Región TRT-MG, se condenó al empleador por discriminación, violación de la dignidad humana, la violación del ejercicio de la función social, y se le obligó al pago de los daños laborales y morales; La mujer no había informado al empresario, su estado de gravidez. E1 Tribunal sostuvo que se violentaron los derechos de la trabajadora apoyado la decisión en el artículo 422 del Código Civil, que aboga por el principio de buena fe en el contrato entre las partes.

La responsabilidad del empleador por los daños depende de la demostración del carácter ilícito de la conducta, el grado de culpabilidad del infractor, en la medida de hecho moralmente perjudicial y la relación causal entre ellos. Cuando se han comprobado estos requisitos, tendrán derecho a una indemnización, y la responsabilidad civil como consecuencia del agente causante del daño (TST - RR: 
14243920135090651, fecha de la sentencia: 11/02/2015, Fecha de publicación: 02/20/2015 DEJT).

La discriminación de la mujer ha tenido que ser protegida igualmente desde el marco legal, con el advenimiento de la redacción de la Ley No 9.779 / 99, que se ocupa de la discriminación en las relaciones laborales en contra de las mujeres, fue aumentado en la consolidación de las Leyes Laborales de la 373-A, este artículo establece las prohibiciones de expresar en los anuncios de trabajo, el sexo, registros corporales de los empleados y actos discriminatorios empleados, los embarazos o de requisitos de prueba de esterilización, así como otras especificaciones descritas en este artículo cuyo propósito es la protección del trabajo de las mujeres.

Con esta legislación se pretendió corregir las distorsiones que afectan el acceso de las mujeres al mercado de trabajo. El artículo 373-A igualmente plantea en el parágrafo único, que estas disposiciones de protección a la mujer no impiden la adopción de medidas encaminadas a la creación de políticas de igualdad entre hombre y mujeres, en particular las destinadas a corregir las distorsiones que afectan a la formación profesional, el acceso al empleo y las condiciones generales de trabajo de las mujeres.

Para André Luiz Paes de Almeida (2014), los derechos destinados a la protección del trabajo de las mujeres comenzaron en el siglo XIX con la Revolución Industrial, porque la mano de obra femenina es muy inferior a la de los hombres, y su deseo de permanecer en el empleo las hacía más vulnerables; Las mujeres terminaron por aceptar la diferencia, llegando a ser las preferidas por los empleadores, en este periodo de tiempo las condiciones higiénicas y de tiempo, largos turnos, no era reglamentada, quedando incluso en ausencia de medidas de protección en tiempos de gestión, lo que provocó la necesidad de legislar a favor de los derechos de las mujeres.

Otavio Lopes (1998) considera que las partes que más sufren la discriminación, tanto en el sentido amplio como la restricción de las relaciones laborales y el empleo, son las mujeres y los negros, que sufren tanto la diferenciación de sexo, color, y las condiciones de equiparación económica y social, el autor también apunta a la fragilidad de esta cuestión, a que la discriminación genera pérdidas económicas y repercusiones psicológicas en los discriminados.

Sin embargo, incluso con la existencia de una fuerte legislación preconizadora de la igualdad de derechos entre hombres y mujeres, la realidad sigue siendo una imagen muy dramática para las mujeres en el lugar de trabajo. Las tradiciones 
culturales, las relaciones económicas, las condiciones de participación en la vida política y social, así como la eficacia del sistema de regulación y la característica de comunicaciones, para confirmar la continuidad de la discriminación. Esta discriminación se presenta en Brasil, con una mayor discriminación en las regiones del noreste y norte, donde la desigualdad socioeconómica y cultural es más evidente.

\subsection{Evidencia de discriminación de la mujer en Colombia}

Se hace necesario realizar un estudio más detallado de la situación que afronta la mujer en Colombia, debido a que Colombia es una de los países con mayor proyección económica de Latinoamérica, establecida junto a Brasil como las economías emergentes más importantes de la zona.

La situación de la mujer en Colombia es similar al sufrido en la región, la tasa de desempleo fue del 11.7\% en tanto que para los hombres apenas llego al 6.8\% para el periodo del trimestre abril-junio del 2015 (DANE, 2015). Por otro lado se estableció que el $56.4 \%$ de las mujeres inactivas se dedicó a oficios del hogar, lo que representará en un futuro una discriminación y pobreza en la vejez para las mujeres.

\subsubsection{Marco normativo de no discriminación}

En la Constitución de 1991 se establece que Colombia se constituirá en un Estado Social de Derecho, con respeto a la dignidad humana. Estableciendo en el artículo 13 el derecho a la libertad e igualdad de todas las personas ante la Ley. Determina igualmente que todos los ciudadanos tienen los mismos derechos, libertades y oportunidades sin ninguna discriminación por razones de sexo, raza, origen nacional o familiar entre otros.

Pero la constitución igualmente plantea que la mujer y el hombre tienen los mismos derechos y oportunidades, estableciendo que la mujer no podrá ser sometida a ninguna clase de discriminación.

El artículo 43 plantea una protección especial a la mujer al establecer que: "Durante el embarazo y después del parto gozará de especial asistencia y protección del Estado, y recibirá de éste subsidio alimentario si entonces estuviere desempleada o desamparada. E1 Estado apoyará de manera especial a la mujer cabeza de familia" (Constitución Política de Colombia, 1991).

En el ámbito laboral el Código Sustantivo del Trabajo colombiano (1950) en el artículo 10 modificado por la Ley 1496 de 2011, establece igualmente, la condición 
de igualdad entre todos los trabajadores, determinando que queda abolida toda forma de distinción jurídica entre trabajadores.

Con la determinación constitucional y el código sustantivo del trabajo se establece de manera adecuada la relación de igualdad de hombre y mujeres en el trabajo. Pero esto no ha sido suficiente para que esta igualdad sea real y efectiva. Por lo que se hizo necesario, para los cargos decisorios en el gobierno y debido a la poca participación de la mujer en los mismos, establecer una ley de cuotas.

Estas acciones afirmativas, que si bien es cierto no son suficientes, han hecho que la mujer pueda acceder a dichos cargos determinando como cuota correspondiente a la mujer un 30\% (Ley 581, 2000).

Por otro lado y reafirmando el derecho a la no discriminación de la mujer en la retribución salarial, se estableció la Ley 1496 del 2011, cuya finalidad es garantizar la igualdad salarial y la retribución laboral entre mujeres y hombres, y se establecen mecanismos para erradicar cualquier forma de discriminación.

Un aspecto importante a resaltar dentro de la Ley 1496 del 2011 es la definición que establece la norma de discriminación en materia de retribución laboral por razón del género o sexo, determinando en el artículo 30. "Discriminación directa en materia de retribución laboral por razón del género o sexo: Toda situación de trato diferenciado injustificado, expreso o tácito, relacionado con la retribución económica percibida en desarrollo de una relación laboral, cualquiera sea su denominación por razones de género o sexo”.

Sin lugar a dudas es un paso importante desde el punto de vista legal en el reconocimiento de una igualdad salarial, pero aun existiendo norma, la brecha salarial persiste en Colombia, en el 2013 giraba alrededor del 21\% según datos suministrados por el mismo Ministerio de Trabajo (2013).

La multiculturalidad de Colombia no reconocida hasta la constitución de 1991 ha hecho que por mucho tiempo no se reconociera el derecho a ser diferente, y no se reconociera la diversidad, lo que conlleva a que los actos discriminatorios fueran evidentes, negros, indios, mulatos, blancos, homosexuales, o mujeres sufrían los condicionamientos de discriminación.

Se hace necesario establecer un marco normativo de carácter penal para quienes ejercieran actos discriminatorios o de racismo, por lo que se establece con la Ley 1482 de 2011 el delito de actos de racismo o discriminación- modificando el artículo 134a del Código Penal así:. "Actos de Racismo o Discriminación. El que arbitrariamente impida, obstruya o restrinja el pleno ejercicio de los derechos de las 
personas por razón de su raza, nacionalidad, sexo u orientación sexual, incurrirá en prisión de doce (12) a treinta y seis (36) meses y multa de diez (10) a quince (15) salarios mínimos legales mensuales vigentes".

Lo anterior protege sin lugar a dudas a la mujer, quien en muchos casos se ve doblemente discriminado como mujer y como indígena o como afrocolombiana.

Dentro del marco penal y como protección especial de la mujer, se ha establecido la Ley 1761 de 2015 donde se establece y se tipifica el feminicidio como un delito autónomo, para garantizar la investigación y sanción de las violencias contra las mujeres por motivos de género y discriminación, así como prevenir y erradicar dichas violencias y adoptar estrategias de sensibilización de la sociedad colombiana, en orden a garantizar el acceso de las mujeres a una vida libre de violencias que favorezca su desarrollo integral y su bienestar, de acuerdo con los principios de igualdad y no discriminación.

\subsubsection{Discriminación de la mujer en ámbito laboral en Colombia}

Como se evidencia del recuento normativo, existen normas sobre no discriminación a la mujer en el ámbito laboral, pero aún persisten actividades de discriminación, lo que ha hecho que la Corte Constitucional, a través de su jurisprudencia, proteja el derecho de muchas mujeres que han sido víctimas de discriminación. Dentro de las sentencias a resaltar se encuentra la T-071 del 2007, T-1002 de 1999, T-873 del 2005, por medio de las cuales se evidencia la discriminación en el acceso al empleo o en la permanencia en el mismo, al exigírsele prueba de embarazo. La Corte Constitucional, como reprochable, plantea que esta conducta implica una grave vulneración de los derechos a la intimidad, el libre desarrollo de la personalidad y el trabajo de las empleadas, al respecto propone:

\footnotetext{
Así, la exigencia de "pruebas de embarazo" por parte de una empresa, con el propósito de condicionar el ingreso o la estabilidad de la trabajadora en la nómina de la misma, es una conducta reprochable que implica vulneración del derecho a la intimidad de la empleada y de su familia y que lesiona también el libre desarrollo de su personalidad, afectando por contera el derecho al trabajo. (Corte Constitucional, 2007)
}

En otras sentencias como en la C-410 de 1994, la Corte Constitucional hace evidente la discriminación que sufre la mujer, planteando que el sexo es el primer 
motivo de discriminación social, porque por lo menos la mitad del conglomerado humano está constituido por mujeres. Por otro lado la corte manifiesta en la mencionada sentencia: “[...] las consecuencias de la diferenciación injustificada por razón de sexo se extienden a insospechados espacios, lo que da cuenta de la naturaleza velada o encubierta de un sinnúmero de prácticas inequitativas que trascienden las manifestaciones más comunes de la discriminación”.

En el mismo fallo, la Corte Constitucional (1994) plantea la discriminación que sufre la mujer en la vida laboral, deteniéndose a analizar que muchas de las labores de las mujeres son labores secundarias mal remuneradas, sin prestaciones sociales, ni seguridad social. Igualmente evidencia que la mujer es quien desarrolla el trabajo doméstico, y las labores como ama de casa, actividades poco valoradas en la sociedad, y no consideradas como trabajo, estos obstáculos afectan el camino hacia la igualdad real y efectiva, ya que las mujeres se encuentran discriminadas no solo por su condicionamiento biológico sino también por la estructura social.

\section{Contribuciones y propuestas para una igualdad real entre mujeres y hombres}

Las condiciones de las mujeres han sido luchas constantes frente al reconocimiento de los derechos humanos, sobre todo el derecho a la igualdad, pero se requiere una igualdad real y efectiva. Desde 1945 hasta la recomendación 27 de la CEDEW en 2010 se ha luchado por la igualdad y no discriminación pero hasta la fecha no se ha conseguido.

La pregunta sería ¿cómo hacer para que la igualdad entre hombres y mujeres sea real? Habrían múltiples respuestas, una primera seria hacer conscientes de su situación a las mujeres, de un empoderamiento de ellas desde su condición de ser mujer, al ser conscientes educarían a sus hijos con criterios de respeto e igualdad frente a la mujer y el respeto a sus derechos humanos.

Una segunda consideración sería una política pública de educación que genere lineamientos educativos en pro de la enseñanza, defensa y respeto de los derechos de la mujer en condiciones de igualdad.

Otro aspecto es el empoderamiento de las mujeres en cargos de representación, que permita hacer cambios más profundos desde los lineamientos democráticos de cada Estado, el hecho que en la región hayan llegado al poder mujeres como Dilma Rouseff (Brasil), Cristina Fernández (Argentina), Laura Chinchilla (Costa 
Rica), Michelet Bachelet (Chile) es un avance en la participación efectiva de las mujeres en el poder.

Sumado a lo anterior, se requiere que sean reconocidas las labores del hogar, de las cuidadoras dentro de los Estados, porque ellas son el fundamento de la construcción de la sociedad. Reconocer beneficios en seguridad social, para poder acceder a una pensión de vejez o reconocer una contraprestación a su trabajo, será dignificar el trabajo de la mujer.

Solo se requiere una igualdad efectiva y real, que se pueda percibir en cada una de las mujeres, quienes desde 1945 tienen derechos humanos consolidados y reconocidos por los organismos internacionales.

\section{Conclusiones}

Se puede evidenciar en el presente documento que los Derechos Humanos de la mujeres y su no discriminación han sido establecidos desde los inicios de la ONU, la misma declaración Universal de los Derechos Humanos (1945) consagra la no discriminación de la mujer; Igual respeto a los derechos de la mujer se plantea en los Pactos de Derechos Civiles y Políticos, y en el Pacto de Derechos Económicos, Sociales y Culturales. Pero es en la Convención sobre la Eliminación de todas las formas de discriminación contra la mujer, donde se dan los lineamientos más específicos sobre los derechos humanos de la mujer. Igualmente se constató la OIT, que a través de los Convenios ha generado el reconocimiento del derecho a la igualdad y no discriminación de la mujer frente a las condiciones laborales.

Pero al analizar la mujer dentro del contexto laboral se pudo evidenciar que a pesar de existir normas internacionales de reconocimiento a los derechos de igualdad y no discriminación a la mujer la realidad es diferente. Solo acceder a un puesto formal genera una brecha laboral, ya que pueden acceder a los puestos de trabajo más hombres que mujeres, siendo un promedio en mujeres para el 2010 del $52.62 \%$ en tanto que para los hombres está en 79.6\%. Pero aun existiendo trabajo formalizado, el valor que se paga en salarios por el mismo trabajo a hombre y a mujeres es diferente, pagando salarios más bajos a las mujeres siendo un promedio del $36 \%$ más bajo.

Por otro lado se evidenció que las mujeres engruesan la masa población dedicada a labores informales o trabajo de servicio doméstico, donde no se reconocen 
adecuadamente los mínimos derechos laborales, y los salarios llegan a ser menores que los salarios reconocidos como mínimos en los países Latinoamericanos. Pero también se pudo constatar que existen trabajos como el de cuidadoras, en los cuales no se le hace a la mujer ningún reconocimiento de tipo económico, ni prestacional.

Se estudió igualmente las consecuencias adversas que existen para las mujeres el tener salarios bajos, trabajos informales, o poco remunerado o no remunerados. Las consecuencias son una gran brecha salarial, una no adecuada cotización en pensiones lo que lleva a que no accedan a una pensión de vejez y la situación de vulnerabilidad y pobreza al no tener recurso para sostenerse en la vejez.

Sin lugar a dudas con este estudio se pudo constatar la hipótesis que se planteó al comienzo; Aun cuando hay normas que regulan el derecho humano a la no discriminación por razones de sexo en Latinoamérica, en el trabajo se sigue discriminando a la mujer por el solo hecho de ser mujer.

\section{Referencias}

Almeida, A. (2014). CLT e Súmulas do TST comentada / André Luiz Paes de Almeida. 11. ed. São Paulo: Rideel. (Série Descomplicada).

Bacila, C. (2005). Estigmas: um estudo sobre os preconceitos. Rio de Janeiro: Lúmen Júris. Banco Mundial. (2014). La mitad de los trabajadores de América Latina tienen un empleo informal. Recuperado de http://www.bancomundial.org/es/news/feature/2014/04/01/ informalidad-laboral-america-latina

CEDAW. (2010). Recomendaciones generales aprobadas por el Comité para la Eliminación de la Discriminación contra la Mujer. Recuperado de http://conf-dts1.unog.ch/1\%20 SPA/Tradutek/Derechos_hum_Base/CEDAW/00_4_obs_grales_CEDAW.html

CEPAL. (2015). CEPAL plantea reconocer trabajo de cuidados no remunerado en la región. Recuperado de http://www.cepal.org/es/noticias/ cepal-plantea-reconocer-trabajo-de-cuidados-no-remunerado-en-la-region

CEPAL, FAO, ONU Mujeres, PNUD y OIT. (2013). Informe Regional. Trabajo decente e igualdad de género. Políticas para mejorar el acceso y la calidad del empleo de las mujeres en América Latina y el Caribe. Chile: ONU Recuperado de http://www.ilo.org/wcmsp5/groups/ public/@americas/@ro-lima/@sro-santiago/documents/publication/wcms_233161.pdf ISBN 978-92-2-328105-2

Código Sustantivo del Trabajo. (1950). Decreto 2663 de 1950 Nivel Nacional. Recuperado de http://www.alcaldiabogota.gov.co/sisjur/normas/Norma1.jsp?i=33104 
Constitución Política de Colombia. (1991). Constitución Política 1 de 1991 Asamblea Nacional Constituyente. Recuperado de http://www.alcaldiabogota.gov.co/sisjur/ normas/Norma1.jsp?i=4125

Constitución Política de la República Federativa del Brasil. (1988). Brasília: DF: Centro Gráfico. Recuperado de http://www.acnur.org/t3/fileadmin/scripts/doc.php?file=Pdf/0507

Corte Constitucional. (1994). Sentencia No. C-410/94. Recuperada de http://www.corteconstitucional.gov.co/RELATORIA/1994/C-410-94.htm

Corte Constitucional. (2007). Sentencia T-071/07. Recuperada de http://www.corteconstitucional.gov.co/RELATORIA/2007/T-071-07.htm

DANE. (2015). Informe de estadísticas laborales. Bogotá.: Departamento Administrativo Nacional de Estadística.

Delgado, M. (2010). Curso de Direito do Trabalho, 9. ed. São Paulo: Revista dos Tribunais. Jiménez, D. (s.f). La mujer en la Legislación de los sistemas de pensiones reformados en América Latina. CEPAL Recuperado de http://www.cepal.org/mujer/proyectos/pensiones/ publicaciones/word_doc/daniel_jimenez.pdf

Lei no 9.029 (Brasil). 13 de abril de 1995. Recuperado de http://www.planalto.gov.br/ ccivil_03/LEIS/L9029.HTM

Ley 581. Diario Oficial No. 44.026. Bogotá, Colombia, 31 de mayo de 2000. Recuperado de http://www.alcaldiabogota.gov.co/sisjur/normas/Norma1.jsp?i=5367

Ley 1482. Diario Oficial No. 48.270. Bogotá, Colombia, 1 de diciembre de 2011. Recuperado de http://wsp.presidencia.gov.co/Normativa/Leyes/Documents/ley148230112011.pdf

Ley 1496. Diario Oficial 48297. Bogotá, Colombia, 29 de diciembre de 2011. Recuperado de http://www.alcaldiabogota.gov.co/sisjur/normas/Norma1.jsp?i=45267\#2

Ley 1761. Diario Oficial 49565. Bogotá, Colombia, 06 de julio de 2015. Recuperado de http://wp.presidencia.gov.co/sitios/normativa/leyes/Documents/LEY\%201761\%20 DEL\%2006\%20DE\%20JULIO\%20DE\%202015.pdf

Lima, F. (2011). Teoria da Discriminação nas relações de trabalho. Rio de Janeiro: Elsevier.

Lopes, O. (1998). A Questão da Discriminação no Trabalho. Recuperado de https://revistajuridica.presidencia.gov.br/index.php/saj/article/viewFile/981/966

Menchu, R. (2002). La Mujer en el siglo XXI. Recuperado de http//www.redceres.org

Ministerio de Trabajo. (2013). La penosa brecha laboral entre hombres y mujeres. Recuperado de http://www.mintrabajo.gov.co/medios-abril-2013/1740-la-penosa-brechalaboral-entre-hombres-y-mujeres.html

OEA. (2015). Por los derechos de las mujeres y la igualdad de género en las Américas. Recuperado de http://www.oas.org/es/cim/docs/FolletoCIM[Jun2015]-ES.pdf 
OCDE, BID y Banco Mundial. (2015) Panorama de las Pensiones: América Latina y el Caribe. Organización de Cooperación y Desarrollo Económicos Recuperado de https://publications.iadb.org/bitstream/handle/11319/6892/Panorama_de_las_Pensiones_America_Latina_y_el_Caribe.pdf ISBN978-1-59782-214-5

OISS. (2014a). Boletin del programa Iberoamericano de Cooperación sobre Adultos Mayores. Jiménez, Adolfo. Madrid: OISS. Recuperado de http://www.oiss.org/IMG/pdf/BOLETIN_No_3.pdf

OISS. (2014b). Curso Mujer y Envejecimiento: Modulo 1: Situación de las mujeres mayores en los paises iberoamericanos. Alonso, José. Madrid: OISS. Recuperado de http://www.oiss. org/IMG/pdf/BOLETIN_No_3.pdf

OIT. (1981). C156 - Convenio sobre los trabajadores con responsabilidades familiares, 1981 (núm. 156). Recuperado de http://www.ilo.org/dyn/normlex/es/f?p=NORMLE XPUB:12100:0::NO::P12100_ILO_CODE:C156

OIT. (2010). Igualdade de gênero e raça no trabalho: avanços e desafios / Organização Internacional do Trabalho (p. 216). ISBN: $9789228234763 ; 9789228234770$ (pdf). Organização Internacional do Trabalho; Escritório no Brasil. igualdade de oportunidades no emprego / igualdade de gênero / mulheres trabalhadoras /raça / Brasil. Recuperado de http://www.oitbrasil.org.br/sites/default/files/topic/gender/pub/igualdade_genero_262.pdf

OIT. (2014). La ruta de la formalización en América Latina y el Caribe. Recuperado de http://www.ilo.org/americas/sala-de-prensa/WCMS_245626/lang--es/index.htm

OIT. (2015a). OIT: Los progresos en materia de igualdad de género en el trabajo continúan siendo insuficientes. Recuperado de http://www.ilo.org/global/about-the-ilo/newsroom/news/ WCMS_348063/lang--es/index.htm

OIT. (2015b). Trabajo decente. Recuperado de http://www.ilo.org/global/topics/decentwork/lang--es/index.htm

OIT. (2015c). Informe Mundial sobre Salarios 2014/2015: Salarios y desigualdad de ingresos. Ginebra. Recuperado de http://www.ilo.org/wcmsp5/groups/public/---dgreports/--dcomm/---pub1/documents/publication/wcms_343034.pdf ISBN:978-92-2-328665-1

OIT. (2015d). OIT: 1,7 millones de personas ingresaron a las filas del desempleo en 2015 en América Latina y el Caribe. Recuperado de http://www.ilo.org/americas/sala-deprensa/WCMS_435175/lang--es/index.htm

Valenzuela, M. (2012) Situación del Trabajo doméstico remunerado en América Latina. OIT: 2012. Recuperado de http://www.ilo.org/wcmsp5/groups/public/---americas/---rolima/documents/article/wcms_195947.pdf 